\title{
Establishment of Patient-Derived Pancreatic Cancer Organoids from Endoscopic Ultrasound-Guided Fine-Needle Aspiration Biopsies
}

\author{
Jee Hyung Lee ${ }^{1,2}$, Haeryoung Kim³ ${ }^{3}$ Sang Hyub Lee ${ }^{1}$, Ja-Lok Kư ${ }^{4}$, Jung Won Chun ${ }^{5}$, Ha Young Seo ${ }^{4}$, Soon Chan \\ $\mathrm{Kim}^{4}$, Woo Hyun Paik${ }^{1}$, Ji Kon Ryu ${ }^{1}$, Sang Kook Lee ${ }^{2}$, Andrew M. Lowy ${ }^{6}$, and Yong-Tae Kim \\ ${ }^{1}$ Department of Internal Medicine and Liver Research Institute, Seoul National University Hospital, Seoul National University College \\ of Medicine, ${ }^{2}$ Natural Products Research Institute, Seoul National University College of Pharmacy, ${ }^{3}$ Department of Pathology, Seoul \\ National University Hospital, Seoul National University College of Medicine, ${ }^{4}$ Department of Biomedical Sciences, Korean Cell Line \\ Bank, Laboratory of Cell Biology and Cancer Research Institute, Seoul National University College of Medicine, Seoul, ${ }^{5}$ Center for \\ Liver and Pancreatobiliary Cancer, National Cancer Center, Goyang, Korea, and ${ }^{6}$ Division of Surgical Oncology, Department of \\ Surgery, Moores Cancer Center, University of California, San Diego, La Jolla, CA, USA
}

\section{Article Info}

Received April 11, 2021

Revised July 8, 2021

Accepted August 3, 2021

Published online December 17, 2021

\section{Corresponding Author}

\section{Sang Hyub Lee}

ORCID https://orcid.org/0000-0003-2174-9726

E-mail gidoctor@snuh.org

Ja-Lok Ku

ORCID https://orcid.org/0000-0002-7090-537X

E-mail kujalok@snu.ac.kr

Jee Hyung Lee and Haeryoung Kim contributed equally to this work as first authors.
Background/Aims: Three-dimensional cultures of human pancreatic cancer tissue also known as "organoids" have largely been developed from surgical specimens. Given that most patients present with locally advanced and/or metastatic disease, such organoids are not representative of the majority of patients. Therefore, we used endoscopic ultrasound-guided fine-needle aspiration (EUS-FNA) to collect pancreatic cancer tissues from patients with advanced pancreatic cancer to create organoids, and evaluated their utility in pancreatic cancer research.

Methods: Single-pass EUS-FNA samplings were employed to obtain the tissue for organoid generation. After establishment of the organoid, we compared the core biopsy tissues with organoids using hematoxylin and eosin staining, and performed whole exome sequencing (WES) to detect mutational variants. Furthermore, we compared patient outcome with the organoid drug response to determine the potential utility of the clinical application of such organoid-based assays.

Results: Organoids were successfully generated in 14 of 20 tumors $(70 \%)$ and were able to be passaged greater than 5 times in 12 of 20 tumors (60\%). Among them, we selected eight pairs of organoid and core biopsy tissues for detailed analyses. They showed similar patterns in hematoxylin and eosin staining. WES revealed mutations in KRAS, TP53, CDKN2A, SMAD4, BRCA1, and $B R C A 2$ which were $93 \%$ homologous, and the mean nonreference discordance rate was $5.47 \%$. We observed moderate drug response correlations between the organoids and clinical outcomes in patients who underwent FOLFIRINOX chemotherapy.

Conclusions: The established organoids from EUS-FNA core biopsies can be used for a suitable model system for pancreatic cancer research. (Gut Liver 2022;16:625-636)

Key Words: Pancreatic neoplasms; Endoscopic ultrasound-guided fine-needle aspiration; Organoid; High-throughput nucleotide sequencing; FOLFIRINOX

\section{INTRODUCTION}

Pancreatic cancer is an aggressive malignancy with, ${ }^{1}$ 5 -year survival rate of less than $10 \%{ }^{2-4}$ While the FOLFIRINOX regimen (oxaliplatin, 5-FU, irinotecan, and leucovorin) is relatively more active than prior systemic options, it is associated with a significant toxicity, and most patients with advanced disease do not achieve objective responses. ${ }^{5}$

In the past, research on anti-cancer drugs was most often performed in cell cultures and animal models, but the predictive value for response in the clinic has been low in the case of pancreatic cancer. ${ }^{6}$ Patient-derived xenograft models are also impractical, due to the amount of tissue 
and the time required to generate them. ${ }^{7}$ Recent advancements in cell culture techniques, largely derived from studies of stem cell biology, have led to the development of 3-dimensional cultures (organoids) derived from tumor biopsy tissue from even small samples. ${ }^{8}$ Compared to 2-dimensional cell culture, the 3-dimensional organoid is reported to better recapitulate the molecular characteristics of the original tissue, ${ }^{9}$ and studies have demonstrated fidelity in response/resistance patterns to cancer therapy organoid-based studies, compared to responses in the clinic. ${ }^{10-16}$

Most pancreatic cancer organoids have been developed from surgical specimens, ${ }^{17}$ however, these cannot represent the majority of pancreatic cancer patients, as only $10 \%$ to $20 \%$ of patients with pancreatic cancer are eligible for surgery. ${ }^{18}$ In clinical practice, endoscopic ultrasoundguided fine-needle aspiration (EUS-FNA) has become the procedure of choice for diagnosing pancreatic cancers especially in patients with locally advanced unresectable and metastatic pancreatic cancers. ${ }^{19}$ Though it has recently been shown that the establishment of pancreatic cancer organoids from EUS-FNA samples is feasible, ${ }^{17,20}$ there is limited data regarding the morphologic and genetic fidelity between organoid and patient biopsy tissue in these cases.

Thus, the goals of this study were to; establish patientderived organoids using EUS-FNA material from pancreatic cancer patients and evaluate the correlation between the drug responses of pancreatic cancer organoids and the clinical outcomes of patients who underwent palliative FOLFIRINOX chemotherapy, in order to evaluate its potential utility in the clinic.

\section{MATERIALS AND METHODS}

\section{Patients and study protocol}

The study was approved by the Institutional Ethics Review Board of Seoul National University Hospital (IRB number: H-1712-056-905) and was performed in accordance with the Declaration of Helsinki. Written informed consent obtained from each participant and/or their legal representative, as appropriate. Between January 2017 and December 2017, patients admitted for EUS-FNA of suspected unresectable pancreatic cancer on radiological examination were assessed for eligibility. The inclusion criteria were: (1) age $>19$ years and (2) pathologically diagnosed pancreatic ductal adenocarcinoma. Exclusion criteria were as follows: (1) inability to obtain informed consent or (2) insufficient tumor tissue obtained by one needle pass of EUS-FNA. The following clinical data of the enrolled patients were retrospectively collected from the electronic medical record system: patient demographics (sex and age), tumor characteristics (e.g., size and location of tumor), site of cancer metastasis, chemotherapy regimen, progression-free survival (PFS), and overall survival (OS). PFS represents the duration of the response of the primary drug. OS was defined as the time from diagnosis to death or last follow-up. The data cutoff date was May 30, 2020, and observations were censored at the time of last follow-up.

\section{Endoscopic ultrasound-guided fine-needle aspiration}

All EUS-FNA procedures were performed by a single experienced echoendoscopist (S.H.L.), who has performed

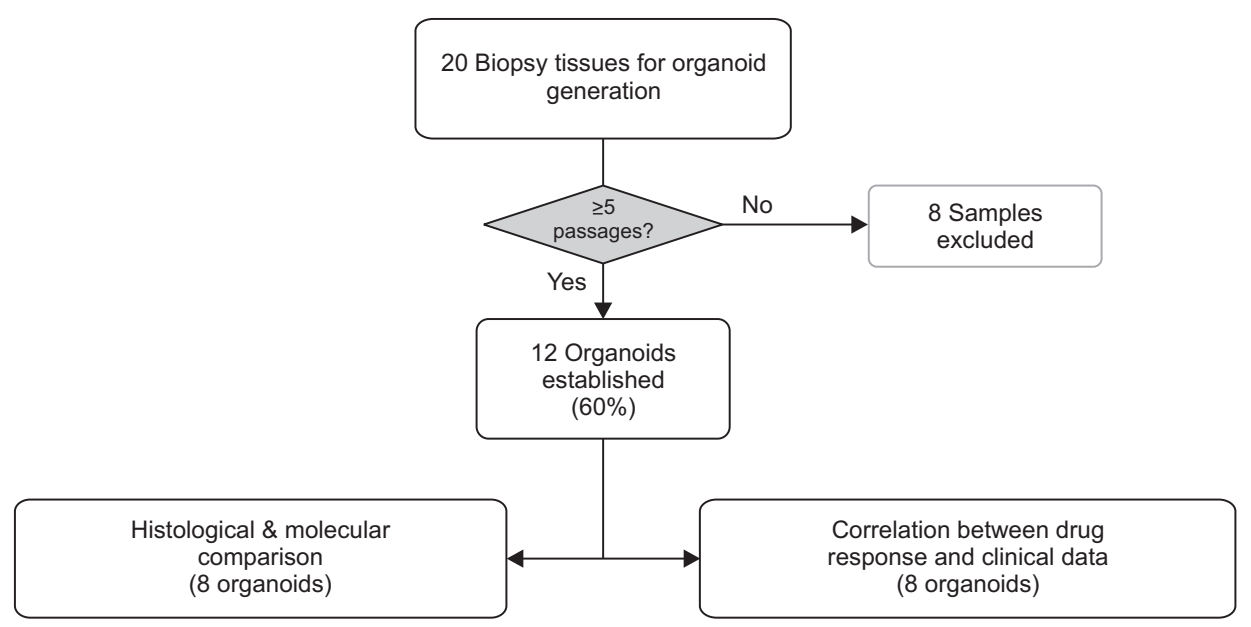

Fig. 1. Study scheme. Among 20 biopsy tissues, 12 organoids were established. Histological \& genetic comparisons (eight organoids); SNU-3947TO, SNU-4158-TO, SNU-4208-TO, SNU-4309-TO, SNU-4354-TO, SNU-4365-TO, SNU-4425-TO, and SNU-4607-TO. Correlation analysis between drug response and clinical data (eight organoids); SNU-4158-TO, SNU-4192-TO, SNU-4206-TO, SNU-4208-TO, SNU-4305-TO, SNU-4354-TO, SNU4365-TO, and SNU-4607-TO. 
more than 100 EUS-FNA procedures annually. A linear EUS scope (GF-UCT260; Olympus Medical Systems, Tokyo, Japan) with a 19- or 22-gauge needle (EZ Shot 3 Plus; Olympus Medical Systems) was used at the discretion of the endoscopist. After visualizing the target lesion in the pancreas by EUS, the needle was punctured into the lesion. Tissue samples for initial diagnosis were obtained through 2 or 3 needle passes with 15 to-and-fro movements, and a $20 \mathrm{~mL}$ suction syringe was applied to acquire adequate tissue. These tissue samples were submitted to the department of pathology for processing of formalin-fixed paraffin-embedded tissue blocks and routine diagnosis. Only a single additional needle pass was performed for the purpose of organoid generation. Tissue samples for research purpose were placed into basal medium (Supplementary Material 1), and immediately transported to the Department of Biomedical Sciences (Korean Cell Line Bank at Seoul National University College of Medicine) for the establishment of pancreatic cancer organoids (Fig. 1).

\section{Establishment of patient-derived organoids}

EUS-FNA biopsy tissues in basal medium were further processed to generate organoids in 24 hours, as shown in Fig. 2. The tissue was washed 3 times with ice-cold Dulbecco's phosphate-buffered saline, and chopped into small pieces of about $2 \mathrm{~mm}$ size on a petri dish. After collecting those pieces in a $50 \mathrm{~mL}$ conical tube, $30 \mathrm{~mL}$ of Dulbecco's phosphate-buffered saline was added, and centrifuged at $1,200 \mathrm{rpm}$ for 5 minutes at $4^{\circ} \mathrm{C}$ (if the collected fractions contained many red blood cells, red blood cell lysis buffer [Sigma-Aldrich, Burlington, MA, USA; R775 7] was added). The fractions were washed until all debris was removed, and the remaining pellet was collected in a 1.5 $\mathrm{mL}$ tube. Matrigel, pre-stored at $4^{\circ} \mathrm{C}$, was added to the 1.5 $\mathrm{mL}$ tube, and mixed with organoid pellet (100 organoids with $50 \mu \mathrm{L}$ Matrigel). After mixing cautiously without making bubbles, $50 \mu \mathrm{L}$ of the mixture was plated onto a 24-well, which was pre-warmed in a $37^{\circ} \mathrm{C}$ incubator. After polymerizing the Matrigel, $500 \mu \mathrm{L}$ of complete medium was added to each well, and the medium in each well was replaced every 3 to 4 days (Supplementary Material 1).

EUS-FNA tissues from 20 patients yielded enough cells to generate organoids. Of the 20 cases, 12 organoids, which had undergone more than 5 passages of growth, were established. Eight organoids (SNU-3947-TO, SNU4158-TO, SNU-4208-TO, SNU-4309-TO, SNU-4354-TO, SNU-4365-TO, SNU-4425-TO, and SNU-4607-TO) had sufficient corresponding biopsy tissues which could be subjected to further molecular analysis (Fig. 1).

\section{Comparison of histological phenotypes between pancreatic cancer biopsy tissues and organoids}

Formalin-fixed paraffin-embedded blocks and hematoxylin and eosin-stained slides of organoids and biopsy tissues were made for the eight organoid-tissue pairs, and the histomorphological features of the organoids and biopsies were compared. Organoids grown in Matrigel for histology were fixed in 10\% paraformaldehyde for 1 hour. The organoids were pre-embedded in Histogel (RichardAllan Scientific HG-4000-012; Thermo Fisher, Waltham, MA, USA), and returned to fixative in $4 \%$ paraformaldehyde overnight. After fixing, the organoids were washed by phosphate buffered saline, and hematoxylin inked. Organoids embedded in Histogel were processed by automated tissue processor (Peloris II; Leica Biosystems, Wetzlar, Germany), and embedded into a paraffin block (Histocentre 3 , Shandon). Samples were sectioned at $4 \mu \mathrm{m}$ (Microtome RM2255; Leica Biosystems) onto poly-l-lysine coated slides, and air-dried at $45^{\circ} \mathrm{C}$ overnight for any subsequent routine hematoxylin-eosin staining. The histology of all tissue and organoid specimens was reviewed independently by a single experienced pancreaticobiliary pathologist (H.K.).

\section{Comparison of genetic homology between biopsy tissues and organoids}

Formalin-fixed paraffin-embedded blocks from eight organoid and tissue pairs were subjected to genomic DNA isolation, whole exome sequencing and nonreference discordance rate (NDR) analysis. The main representative mutations in pancreatic cancer, including KRAS, TP53, CDKN2A, SMAD4, BRCA1, BRCA2, and ARID1A, were compared between the organoids and biopsy tissues. ${ }^{18}$ Paired-end sequences were first mapped to the human genome by HiSeq Instrument, where the reference sequence was UCSC assembly hg19 (original GRCh37 from NCBI, February 2009), using the mapping program BWA (version 0.7.12), and a mapping result file was generated in BAM format using BWA-MEM. Then, Picard-tools (ver.1.130) were applied, in order to remove polymerase chain reaction duplicates. The local realignment process was performed to locally realign reads with BAM files. By using Genome Analysis Toolkit, base quality score recalibration and local realignment around indels were performed. Haplotype Caller of GATK was used for variant genotyping for each sample based on the BAM file previously generated (SNP and short indels candidates were detected). Those variants were annotated by SnpEff v4.1g, to vcf file format, filtering with dbSNP for the version of 142 . Then, SnpEff was applied to filter additional databases, including ESP6500, ClinVar, and dbNSFP 2.9 (Supplementary Mate- 

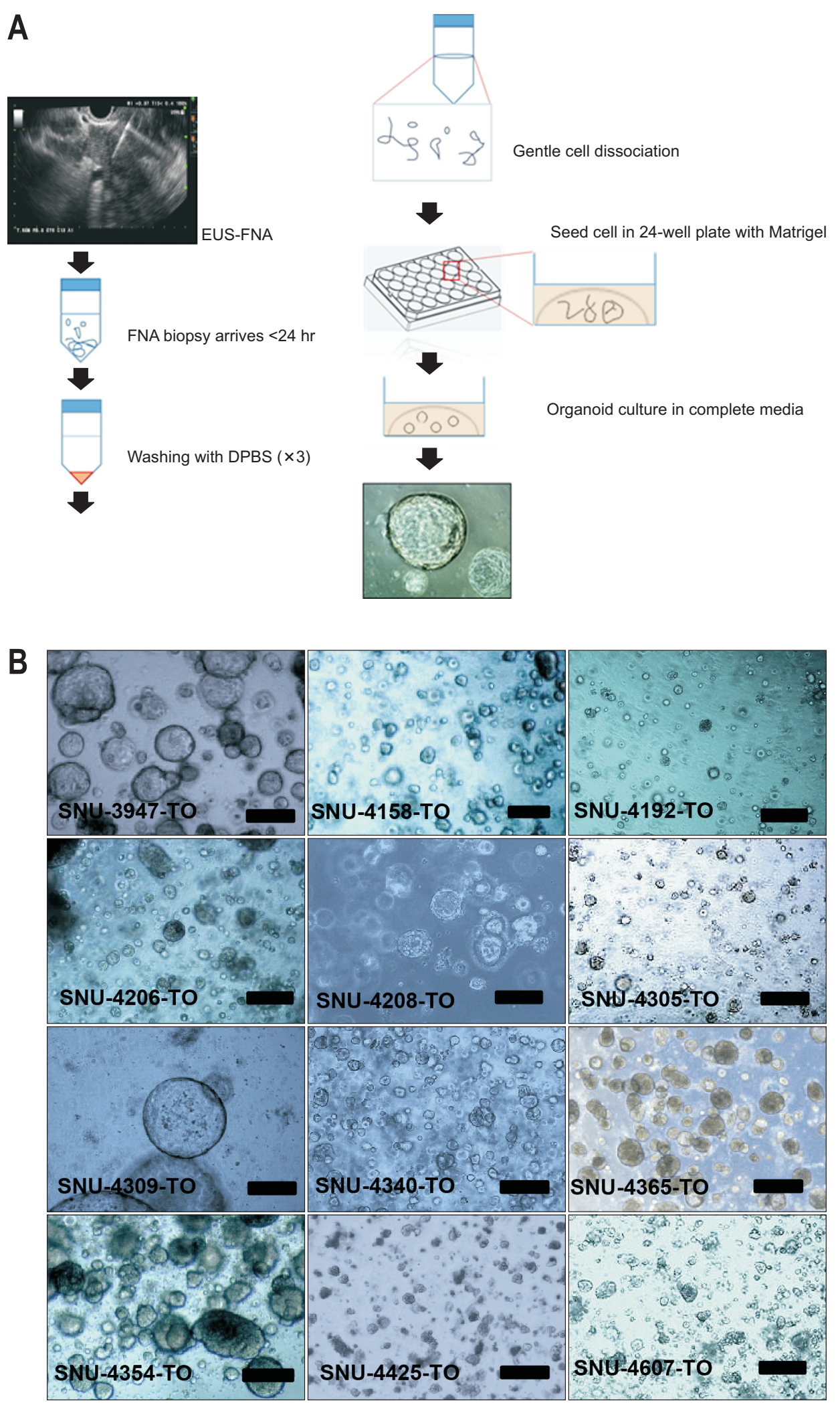

Fig. 2. (A) Organoid generation from endoscopic ultrasound-guided fineneedle biopsy sampling. (B) Brightfield microscopy images of 12 established organoids. Scale bars, $400 \mu \mathrm{m}$.

EUS-FNA, endoscopic ultrasoundguided fine-needle aspiration; DPBS, Dulbecco's phosphate-buffered saline.

rial 2). NDR analysis is a method of calculating the genotype difference in percentage of the biallelic snp position where both two results have variation. This was analyzed by calculating the NDR, as follows:

$\operatorname{NDR}(\%)=\left(\right.$ hom $_{\text {non match }}+$ het $\left._{\text {non match }}\right) /\left(\right.$ hom $_{\text {match }}+$ hom $_{\text {non match }}+$ het $_{\text {match }}+$ het $\left._{\text {not match }}\right) \times 100 \%$ 
NDR of less than $1 \%$ means that two samples considered relatively equal to each other. On the other hand, NDR of about $30 \%$ means that they are relatively different from each other. The lower the NDR, the more similar the two samples are.

\section{Drug response assay of FOLFIRINOX on organoids for clinical correlation}

Among the 12 successfully established organoids, eight organoids (SNU-4158-TO, SNU-4192-TO, SNU-4206TO, SNU-4208-TO, SNU-4305-TO, SNU-4354-TO, SNU4365-TO, and SNU-4607-TO) that grew at similar expansion speed and were derived from patients treated with FOLFIRINOX regimen were selected for drug response assay (Supplementary Table 1, Supplementary Fig. 1A). Organoids were dissociated into single cells, and $600 \mathrm{vi}$ able cells were seeded per well in $50 \mu \mathrm{L}$ (50\% Matrigel/50\% human complete organoid media). ${ }^{21-23}$ Chemotherapeutic agents used in the FOLFIRINOX regimen were added 72 hours after seeding: ${ }^{24}$ oxaliplatin (range, 0.2 to $20 \mu \mathrm{M}$ ), 5-FU (range, 0.02 to $2 \mathrm{mM}$ ), irinotecan (range, 0.3 to 30 $\mu \mathrm{M}$ ), and leucovorin (range, 0.8 to $80 \mu \mathrm{M}$ ), with concentration ratios of $1: 100: 1.5: 4$, respectively. In order to determine the concentration of these chemoagents, we used the standard dose of FOLFIRINOX in clinical practice, as follows: ${ }^{5}$ oxaliplatin $85 \mathrm{mg} / \mathrm{m}^{2}, 5-\mathrm{FU} 2,400 \mathrm{mg} / \mathrm{m}^{2}$, irinotecan $180 \mathrm{mg} / \mathrm{m}^{2}$, and leucovorin $400 \mathrm{mg} / \mathrm{m}^{2}$. We then calculated the molecular concentration of each chemoagent and diluted the final concentration according to the organoid's drug response while sustaining the ratio of each chemoagent. $^{25}$ The final concentrations were serially diluted by $10-$ fold. Compounds were dissolved in dimethyl sulfoxide, and all treatment wells were normalized to $1 \%$ dimethyl sulfoxide. After 3 days, cell viability was assessed by 3D CellTiterGlo according to the manufacturer's instructions (Promega, Madison, WI, USA) on a Luminoskan Ascent (Thermo Fisher) plate reader. All of the experiments were carried out in triplicate, and are represented as mean \pm standard deviation. After having established the FOLFIRINOX concentrations according to the prescriptions for the patients, and applying them to the organoids, dose response curves were generated by luminescence. The area under the curve (AUC) was calculated, and normalized by dividing the AUC value by the maximum area. The normalized AUC range was between 0 and 1 . To determine whether patient-derived organoids reflected the drug sensitivity of the primary tumors, we compared the drug response and the OS with the clinical information of each individual patient.

\section{Statistics}

Continuous variables are presented as means and ranges of values. Categorical variables are reported as proportions (\%). Variables of drug responses are presented as mean \pm standard deviation. The bivariate Spearman's correlation analysis was conducted to describe the association between organoid drug response and survival outcomes. All statistical analyses were conducted with SPSS software version 25.0 (SPSS Inc, Chicago, IL, USA).

\section{RESULTS}

\section{Patient characteristics}

Table 1 shows the overall clinical characteristics of 12 patients from whom organoids were successfully established including; two males, and 10 females (mean age, $61.7 \pm 9.1$ years). Among them, seven patients (58\%) were diagnosed in the metastatic disease with liver, lung, or peritoneal metastases. The size of the primary pancreatic tumors was $(3.8 \pm 1.2 \mathrm{~cm})$. All patients except one had received FOLFIRINOX as first-line chemotherapy. The mean OS was 12 months (range, 1.63 to 34.7 months). Among the 12 successfully established organoids, eight organoids (SNU-4158-TO, SNU-4192-TO, SNU-4206-TO, SNU4208-TO, SNU-4305-TO, SNU-4354-TO, SNU-4365-TO, and SNU-4607-TO) were selected for drug response assay (Supplementary Table 1, Supplementary Fig. 1A). Among the eight organoids, five organoids (SNU-4158-TO, SNU4192-TO, SNU-4208-TO, SNU-4305-TO, and SNU-4365TO) were derived from patients with metastatic (stage IV) disease and three organoids (SNU-4354-TO, SNU-4206TO, and SNU-4305-TO) with locally advanced (stage III) disease.

\section{Establishment of patient-derived organoids from EUS-FNA specimens}

Twenty EUS-FNA specimens that had sufficient cells

Table 1. Baseline Characteristics of the Patients

\begin{tabular}{lc}
\multicolumn{1}{c}{ Characteristics } & Data \\
\hline Sex, male/female & $2 / 10$ \\
Age, mean $\pm S D, y r$ & $61.7 \pm 9.1$ \\
Size, mean $\pm S D$, cm & $3.8 \pm 1.2$ \\
Location, head/body/tail & $5 / 2 / 5$ \\
Needle, 19G/22G & $7 / 5$ \\
TNM staging, III/IV & $5 / 7$ \\
Metastasis site, liver/lung/peritoneum & $8 / 5 / 3$ \\
1st line chemotherapy, No. (\%) & \\
FOLFIRINOX & $11(91.7)$ \\
Gemcitabine \& nab-paclitaxel & $1(8.3)$ \\
Overall survival, mean (range), mo & $12.0(1.6-34.7)$ \\
\hline
\end{tabular}

G, gauge; FOLFIRINOX, oxaliplatin, 5-FU, irinotecan, and leucovorin. 
to generate organoids were used. Organoids were isolated in 14 of 20 tumors (70\%), and organoids with more than five passages of growth were established in 12 of 20 tumors (60\%) (Fig. 1). The established organoids were SNU-3947TO, SNU-4158-TO, SNU-4192-TO, SNU-4206-TO, SNU4208-TO, SNU-4305-TO, SNU-4309-TO, SNU-4340-TO, SNU-4354-TO, SNU-4365-TO, SNU-4425-TO, and SNU4607-TO (Fig. 2B). All 12 organoids underwent over 20 passages of growth.

\section{Morphological similarity between biopsy tissues and organoids}

Eight pairs of biopsy and their corresponding organoids were compared for their histomorphological characteristics. We found similarities in the morphology of the tumors in the biopsy specimens and corresponding organoids, including the architecture (e.g., degree of tubule formation) and cytology (e.g., presence of mucin-containing cells, degree of nuclear pleomorphism) (Fig. 3). This demonstrated that the histology of the organoids recapitulated that of the original tumor.

\section{Similarity in mutational profiles between biopsy tissues and organoids}

The mutational profiles of eight pairs of biopsy specimens and corresponding organoids were compared by whole exome sequencing analysis. The majority of mutational variants present in the biopsy tissues were retained in the corresponding organoids (mean, $89.1 \%$; range, $88 \%$ to $90 \%$ ) (Fig. $4 \mathrm{~A}$ ). Discordance in the mutational profiles between biopsy and organoid pairs were found for less than $12 \%$, suggesting that the organoids represent the mutational profiles of tumors with little bias of different mutations ( $7.36 \%$ on average). The most frequently mutated genes in pancreatic cancer, such as KRAS, TP53, CDKN2A, SMAD4, BRCA, BRCA2, and ARID1A, were mutated in organoids with $93 \%$ homology (Fig. 4B). Concordance in missense KRAS mutations was seen in seven biopsy-organoid pairs, and there was significant enrichment in KRAS G12D mutation in the organoid (Supplementary Fig. 2). Seven pairs of organoids and biopsies showed missense mutations of TP53 R273H and one case showed a frame shift variant (SNU-4425-TO). Missense mutations in BRCA1 and BRCA2 were identified in both pancreatic organoids and corresponding biopsy tissues in four (SNU-3947, SNU-4158, SNU-4309, and SNU-4365) and seven (SNU-3947, SNU-4158, SNU-4208, SNU-4354, SNU-4365, SNU-4425, and SNU-4607) pairs, respectively. We also found concordant frame shift-deletion and insertion mutations in CDKN2A (SNU-3947 and SNU-4309, respectively) and ARID1A (SNU-4425 and SNU-4158, respectively). These results represent that matched biopsy tissue and organoid revealed similar representative mutational variants.

\section{Comparison of genotype difference between biopsy tissues and organoids}

The mean of NDR was 5.47\% (range, 3.16\% to $7.15 \%$ ) (Table 2, Supplementary Table 2). NDRs calculated with each score in Table 2 show that the number of heteronon match (hetero allele in the biopsy, homo allele in the organoid) was relatively higher than the homo-non match

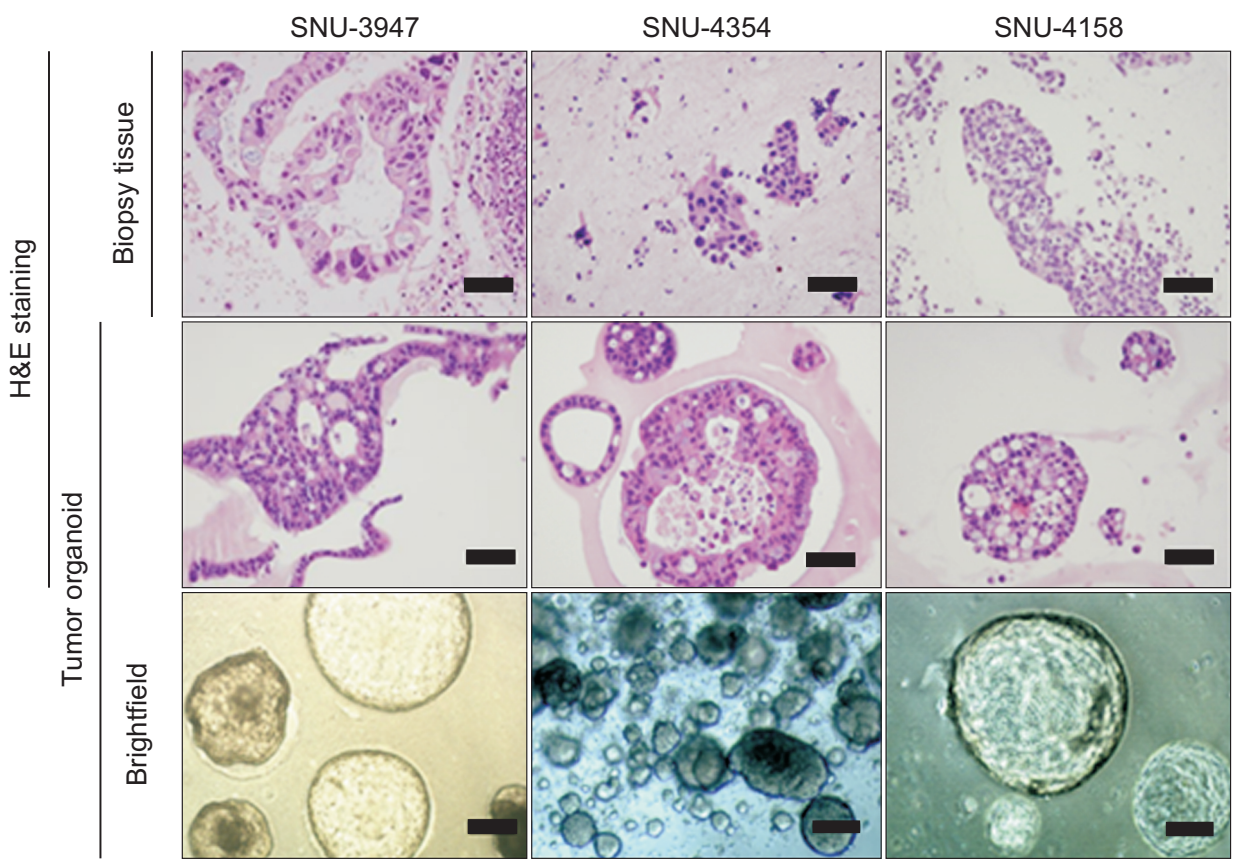

Fig. 3. Representative H\&E staining of patient biopsy tissues and pancreatic cancer-derived organoids and corresponding bright-field microscopy images. Scale bars: $400 \mu \mathrm{m}$. 
A
SNU-3947

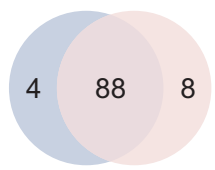

SNU-4354

$\begin{array}{lll}3 & 90 & 7\end{array}$

B
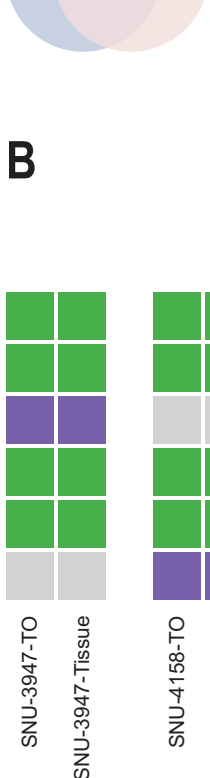

Biopsy
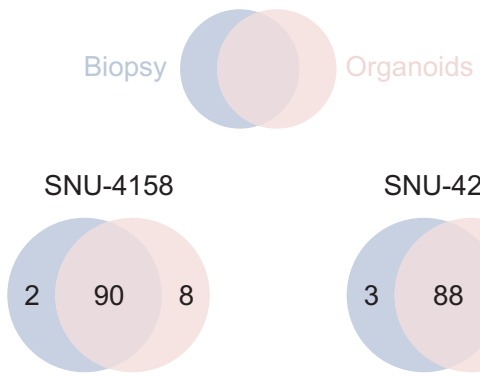

SNU-4365

$\begin{array}{lll}3 & 89 & 8\end{array}$

SNU-4208

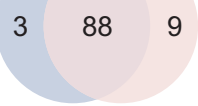

SNU-4425

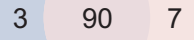

SNU-4309

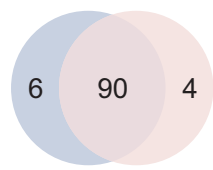

SNU-4607

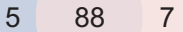

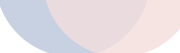
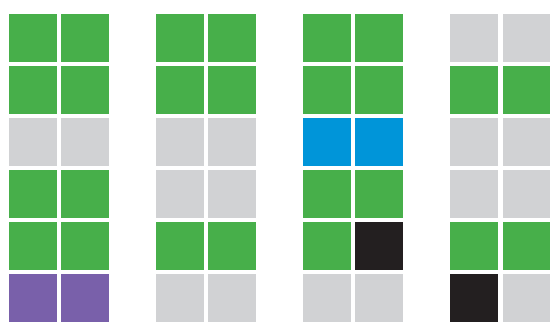

Missense_mutation - Nonsense mutation

- Frame_shift_del Frame_shift_ins

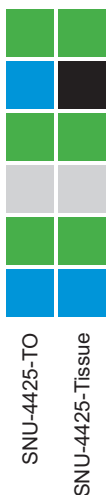

Splice_site

Multi hit

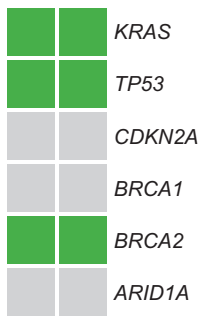

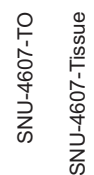

In_frame_del
Fig. 4. (A) Venn diagrams of mutations present in biopsy tissues and organoids. (B) Mutational comparison of organoids and biopsy tissues. Representative mutations: KRAS, TP53, CDKN2A, BRCA1, BRCA2, and $A R I D 1 A$ in an organoid and biopsy tissue pair (SNU3947-TO, organoid; SNU-3947-tissue, biopsy tissue).

Table 2. NDR between Core Biopsy Tissue and Organoid

\begin{tabular}{cccccccc}
\hline Patient & Biopsy tissue & Organoid & Homo-match & Homo-non match & Het-match & Het-non match & NDR \\
\hline SNU-3947 & SNU-3947-T & SNU-3947-TO & 37,703 & 407 & 41,375 & 5,161 \\
SNU-4158 & SNU-4158-T & SNU-4158-TO & 38,252 & 272 & 43,345 & 4,266 \\
SNU-4208 & SNU-4208-T & SNU-4208-TO & 38,464 & 385 & 39,069 & 5,583 \\
SNU-4309 & SNU-4309-T & SNU-4309-TO & 37,192 & 1,208 & 40,724 & 1,336 & 7.15 \\
SNU-4354 & SNU-4354-T & SNU-4354-TO & 38,213 & 381 & 43,184 & 3,665 & 4.74 \\
SNU-4365 & SNU-4365-T & SNU-4365-TO & 38,771 & 277 & 44,183 & 4,438 \\
SNU-4425 & SNU-4425-T & SNU-4425-TO & 39,074 & 220 & 43,149 & 4,742 & 5.38 \\
SNU-4607 & SNU-4607-T & SNU-4607-TO & 38,451 & 1,210 & 39,854 & 3,614 & 5.80 \\
\hline
\end{tabular}

Biopsy tissue and corresponding organoid: homo-match, tumor biopsy's and organoid's allele are homo; homo-non match, tumor biopsy's allele is homo but organoid's allele is hetero; hetero-match, tumor biopsy's and organoid's allele are hetero; het-non match, tumor biopsy's allele is hetero but organoid's allele is homo.

NDR, nonreference discordance rate.

(homo allele in the biopsy, hetero allele in the organoid). According to the results, the number of hetero-non match increased in each sample compared to homo-non match, and subsequently, the NDR was also raised, according to the NDR formula (Table 2). As expected, the NDRs between the different samples were significantly higher

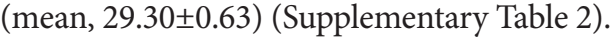

\section{Correlation between drug response of organoid and clinical outcome}

Therapeutic response was assessed on eight patientderived organoids exposed to FOLFIRINOX, a common 
chemotherapeutic regimen for pancreatic cancer (Supplementary Fig. 1B and Supplementary Fig. 1C). Drug responses of the organoids to FOLFIRINOX are illustrated in Supplementary Fig. 1D. FOLFIRINOX exerted a significant suppressive effect on the organoids. By clustering organoid drug response using AUC, the response could be divided into three subgroups: the least responsive (AUC 0.8-1; SNU-4354, SNU-4365, and SNU-4208); intermediately responsive (AUC 0.58-0.76; SNU-4192, SNU-4158, and SNU-4607); and the most responsive (AUC 0.35-0.43; SNU-4305 and SNU-4206) (Table 3). Among the eight patients, two patients, SNU-4607 and SNU-4192 (intermediately responsive group) showed longer than median PFS of 6.1 months. ${ }^{26}$ Of the least responsive group, SNU-4354 and SNU-4365 demonstrated rapid progression (Table 3), and the SNU-4208 was categorized as the most resistant drug response and middle OS of eight patients. In contrast, there were several instances where responses in the organoid were discordant to clinical outcome. SNU-4192 and SNU-4607 were categorized as having an intermediate drug response; however, they showed the longest OS of the eight patients. SNU-4158 was also grouped as intermediately responsive; however, the patient had similar OS to the least responsive group, SNU-4365 and SNU-4354. SNU4206 and SNU-4305 were the most sensitive organoids to FOLFIRINOX; however, the patients' survival was middle of eight patients. The moderate tendency of correlation was found between the organoid's drug response (AUC) and the patient's OS (Spearman correlation coefficient, $\rho O S=0.48)$. We compared PFS and the organoid's AUC after FOLFIRINOX treatment. The result of the agreement between PFS and AUC was lower $(\rho \mathrm{PFS}=0.29)$ than between OS and AUC ( $\rho \mathrm{OS}=0.48$ ). However, we observed a higher degree of agreement when we divided patients according to their cancer stages (stage III $\rho \mathrm{PFS}=1.0$; stage IV $\rho$ PFS $=0.60$ ). Furthermore, the result of the agreement

Table 3. Spearman Correlation Coefficient Analysis

\begin{tabular}{|c|c|c|c|c|c|c|c|}
\hline \multirow{2}{*}{ No. } & \multirow{2}{*}{ Sample } & \multirow{2}{*}{ AUC } & \multirow{2}{*}{ OS, day } & \multicolumn{2}{|c|}{ Sample ranking } & \multirow{2}{*}{$d$} & \multirow{2}{*}{$d^{2}$} \\
\hline & & & & AUC & OS & & \\
\hline 1 & SNU-4354-TO & 0.80 & 70 & 3 & 7 & -4 & 16 \\
\hline 2 & SNU-4158-TO & 0.76 & 76 & 4 & 6 & -2 & 4 \\
\hline 3 & SNU-4607-TO & 0.74 & 839 & 5 & 2 & 3 & 9 \\
\hline 4 & SNU-4305-TO & 0.35 & 214 & 8 & 5 & 3 & 9 \\
\hline 5 & SNU-4365-TO & 0.97 & 49 & 2 & 8 & -6 & 36 \\
\hline 6 & SNU-4206-TO & 0.43 & 349 & 7 & 3 & 4 & 16 \\
\hline 7 & SNU-4208-TO & 1.00 & 252 & 1 & 4 & -3 & 9 \\
\hline 8 & SNU-4192-TO & 0.58 & 1,043 & 6 & 1 & 5 & 25 \\
\hline
\end{tabular}

AUC, area under the curve; OS, overall survival; $d$, deviation; $d^{2}$, standard deviation.

Spearman correlation coefficient ( $r s=0.48$, moderate). Range and strength are: 0-0.2 (very weak), 0.2-0.4 (weak), 0.4-0.6 (moderate), 0.6-0.8 (strong), and 0.8-1.0 (very strong).

\section{A}

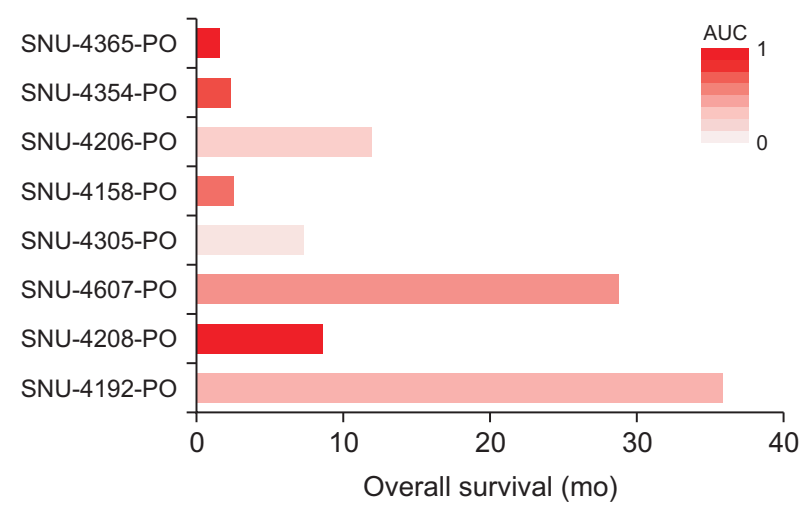

B

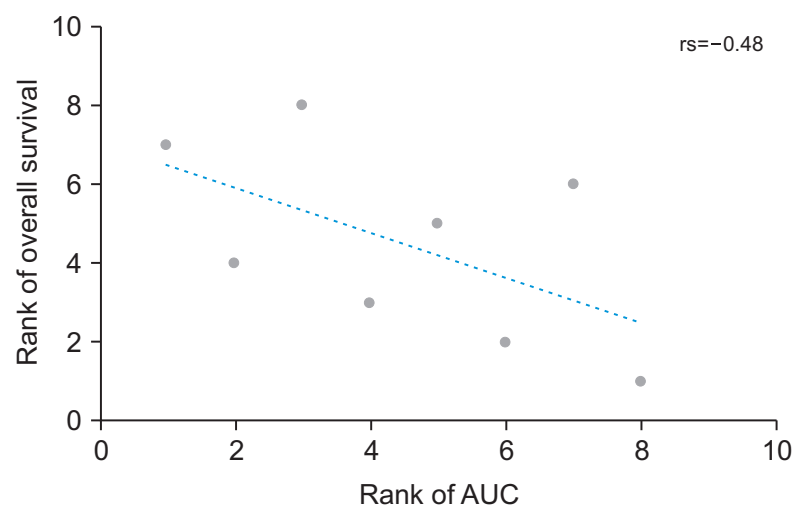

Fig. 5. (A) X-axis, overall survival months of patients who underwent the FOLFIRINOX regimen; Y-axis, overall survival of organoids (SNU-4365-PO, SNU-4365-patient overall survival); the AUCs of eight organoids treated with the FOLFIRINOX regimen are illustrated by heatmap (0<AUC<1). (B) Correlation between patient overall survival and AUC by Spearman's correlation coefficient (rs).

FOLFIRINOX, oxaliplatin, 5-FU, irinotecan, and leucovorin; AUC, area under the curve. 
between PFS and AUC $(\rho \mathrm{PFS}=1.0)$ is higher than between OS and AUC ( $\rho \mathrm{OS}=0.5)$ in stage III. In cases of stage IV, $\rho \mathrm{PFS}$ and $\rho \mathrm{OS}$ were 0.6 and 0.7 , respectively. The result of organoid's drug response on each chemotherapeutic agent does not seem to correlate well with clinical outcomes (Table 3, Fig. 5, Supplementary Table 3).

\section{DISCUSSION}

Pancreatic cancer is a highly drug resistant malignancy and the etiology of this resistance remains obscure but is likely multifactorial in nature. Hence, treatment with a single standardized regimen is not likely be successful. ${ }^{27}$ In order to prescribe a personalized drug to each individual patient, ideally a model that mimics the behavior of the patient's tumor could be developed and rapidly evaluated. The organoid is a model of intense interest as it is derived directly from the patient's cancer and can be propagated. ${ }^{9}$ Furthermore, the organoid can be developed by means of EUS-FNA and thus can theoretically be used to evaluate all pancreatic cancer patients, regardless of stage. ${ }^{24}$ In this study, we evaluated whether organoids derived from EUSFNA biopsy tissue represent pancreatic cancer tissue with respect to histology and genetics, as well as the established organoid's drug response relative to the patient's clinical outcome. We showed a high degree of mutational concordance and histological similarity between biopsy tissue and organoid, and also suggested correlation between the patient's clinical data and the organoid drug (FOLFIRINOX chemotherapy) response.

In the previous studies, Boj et al. ${ }^{8}$ first reported the establishment of patient-derived pancreatic cancer organoids. However, the majority of these organoids were established from surgically-resected pancreatic cancer tissues, and thus represent only the small portion of pancreatic cancer patients who are candidates for surgery. Tiriac et al. ${ }^{17}$ reported on organoids developed from EUS-FNA biopsies, which can represent patients who cannot be operated on surgically. They showed an isolation rate of organoid of $87 \%$, and an establishment rate of $66 \%$. Recently, Lacomb et al. ${ }^{20}$ reported the number of biopsy passes for EUS-FNA sampling did not affect the yield of organoid generation, and that failure of the establishment in all specimens is likely due to a high number of epithelial cells, which arrest the proliferation of organoid. In our study, we established organoids by means of EUS-FNA and the establishment rate was $60 \%$, which is relatively low, compared to the previous study. ${ }^{17}$ The discrepancy of the organoid success rate may have resulted from the difference in procedural details for acquiring tissue samples. Various factors influence the yield of EUS-guided tissue acquisition, such as the needle gauge and type, the use of stylet and suction, the application of fanning technique, tumor size and location, and the experience of the echoendoscopists. ${ }^{28}$ We obtained the samples using a suction syringe, whereas the slow pull technique was performed in two other studies. ${ }^{17}$ Rapid onsite evaluation was not available at our hospital, therefore, more needle movements were required to acquire adequate tissues. These factors may result in cell damage and contamination from blood or epithelial cells. The needle type was also different: the aspiration and biopsy combined needle in this study; meanwhile, biopsy needle (SharkCore FNB Exchange System; Medtronic Inc., Minneapolis, MN, USA) or Acquire FNB Device (Boston Scientific, Marlborough, MA, USA) in other studies. ${ }^{17}$ Finally, a learning curve effect may also affect the whole sequences of organoid generation (sample acquisition, processing a sample, creation and growth of organoids). In general, an organoid culture is established when the organoid lines have been consecutively passaged more than 5 times. Although there are critical variations depending on the size of the biopsy tissue, it typically takes about 2 to 3 months to successfully generate a stable culture and sufficient organoid numbers for drug screening. However, we only applied FOLFIRINOX, thus reducing the time to approximately 1 to 2 months, with some interpatient variations. Therefore, when the organoid is applied for the estimation of clinical result in each patient for precision medicine, it would be useful to narrow down the number of drugs based on the molecular and genetic aspects of the patient. Although improvements are needed in various aspects to overcome obstacles to direct application as personalized precision medicine, the major strength of drug screening using patient-derived organoids is that it can be performed in parallel for numerous anti-cancer drugs after the establishment of a stable culture. This allows for efficient decision-making in the selection of a second-line treatment, particularly in case of nonresponse for standard first-line treatment.

We compared the morphological aspects of the tumor between biopsy tissue and its corresponding organoid, and observed striking similarities. Our histological analysis demonstrated that pancreatic cancer patient-derived organoids retained the major characteristics of the tumor biopsies, concordant with previous studies. ${ }^{29,30}$

In this study, we confirmed that the established organoids displayed genetic alteration highly concordant with those is in the tumor biopsy. The results indicated that the organoid from EUS-FNA retained about $93 \%$ of the most common mutations (KRAS, TP53, CDKN2A, BRCA1, $B R C A 2$, and $A R I D 1 A$ ) present in the original pancreatic 
cancer biopsy, and showed high mutational concordance rate, on average, $89.1 \%$, which is similar to Mapes et al.'s observation $^{31}$ that the somatic variant concordance between primary tumors and corresponding organoids resulted in an average $88.1 \%$, in 12 cancer types. Tiriac et al. ${ }^{32}$ observed $97.43 \%$ (range, $82.49 \%$ to $99.96 \%$ ) concordance between patient-derived organoids derived from surgical resections and their corresponding primary tumors (both of them were germline corrected using normal tissue) in pancreatic cancer, and Broutier et al., ${ }^{33}$ on average, 92\% mutational concordance in liver cancer. In this study, we used NDR to observe genetic variants, and found that our NDR (mean, $5.47 \%$; range, $3.16 \%$ to $7.15 \%$ ) is acceptable, considering the range of confidence (higher than 30\% means different sample from each other, while lower than $1 \%$ means the same origin). The most likely explanation for discordant findings are that as the organoid is cultured it may accumulate additional mutations beyond those that existed in the original tissue. This is strongly supported by in Supplementary Fig. 1, where the KRAS exon2 mutations were enriched in organoid, compared to the original tissue. Therefore, as indicated in Table 2, het-non match (tumor biopsy's allele is hetero, but organoid's allele is homo) is higher than homo-non match (tumor biopsy's allele is homo, but organoid's allele is hetero). This phenomenon was also illustrated in the previous study, in which organoids were continually cultured and sequenced at different passages to observe clonal dynamics; mutated KRAS in subclone, which became the dominate clone in culture. ${ }^{34}$

A clinical trial examining the establishment of pancreatic cancer organoids developed from EUS-guided fine-needle biopsy has been conducted (ClinicalTrials. gov Identifier: NCT03140592). Furthermore, drug screening of pancreatic cancer organoids developed from EUSFNA tissues are being tested (ClinicalTrials.gov Identifier: NCT03544255). Driehuis et al. ${ }^{24}$ showed an overall correlation between patient clinical data and organoid drug response using gemcitabine in pancreatic cancer, while Tiriac et al. ${ }^{32}$ also showed similar results in pancreatic cancer studying five chemotherapeutic agents (gemcitabine, nab-paclitaxel, irinotecan, 5-FU, and oxaliplatin). However, until now, there are few reports that have considered current clinical practice using FOLFIRINOX chemotherapy in the management of advanced pancreatic cancer patients. In our study, we showed moderate correlation between the established organoid's drug response, and the patient OS pattern. We compared PFS and the organoid's AUC after FOLFIRINOX treatment. The result of the agreement (between PFS and AUC) was lower than that of OS ( $\rho \mathrm{PFS}=0.29$ and $\rho \mathrm{OS}=0.48$ ). However, we observed a higher degree of agreement when we divided patients according to their cancer stages (stage III $\rho \mathrm{PFS}=1.0$, stage IV $\rho \mathrm{PFS}=0.60$ ). This result suggests that the resistance therapeutic profiling of organoids would be potentially relevant to the clinical course of the individual patient. However, a selection bias can be occurred by these analyses due to the small number of patients and uncontrolled clinical variables in nature of retrospective data. ${ }^{35,36}$

Our study has other limitations as retrospective, single center study, with a relatively small sample size. However, this study further contributes to the current body of literature, as these organoids were established from the EUSFNA biopsies from patients with advanced pancreatic cancer. We were able to assess the molecular, histologic and drug sensitivity of the organoids and compare the latter to the outcomes of patients treated with FOLFIRINOX standard of care chemotherapy.

In conclusion, this study demonstrated the feasibility of establishing patient-derived organoids using EUS-FNA in advanced pancreatic cancer patients. We observed moderate correlations between the organoid drug responses and patient clinical outcomes. These results serve as a modest foundation for future studies to evaluate the role of organoids in developing personalized approaches to the treatment of pancreatic cancer.

\section{CONFLICTS OF INTEREST}

J.K.R. is an editorial board member of the journal but was not involved in the peer reviewer selection, evaluation, or decision process of this article. No other potential conflicts of interest relevant to this article were reported.

\section{ACKNOWLEDGEMENTS}

This work was supported by the Seoul National University Hospital Research Fund (grant number: 05-20170040).

\section{AUTHOR CONTRIBUTIONS}

Conceptualization: S.H.L., J.L.K. Data curation: J.H.L., H.K., J.W.C. Formal analysis: J.H.L., H.K. Funding acquisition: S.H.L. Methodology: H.Y.S., S.C.K. Project administration: W.H.P., J.K.R., S.K.L., A.M.L., Y.T.K. Visualization: J.H.L., H.K., J.W.C., H.Y.S., S.C.K. Writing - original draft: J.H.L. Writing - review \& editing: J.H.L., H.K., J.W.C., S.H.L., J.L.K. Approval of final manuscript: all authors. 


\section{ORCID}

Jee Hyung Lee

https://orcid.org/0000-0002-0580-5022

Haeryoung Kim

https://orcid.org/0000-0002-4205-9081

Sang Hyub Lee

Ja-Lok Ku

Jung Won Chun

Ha Young Seo

Soon Chan Kim

Woo Hyun Paik

Ji Kon Ryu

Sang Kook Lee

Andrew M. Lowy

Yong-Tae Kim

https://orcid.org/0000-0003-2174-9726

https://orcid.org/0000-0002-7090-537X

https://orcid.org/0000-0003-1964-7501

https://orcid.org/0000-0001-6063-9680

https://orcid.org/0000-0002-7969-4804

https://orcid.org/0000-0001-8708-3280

https://orcid.org/0000-0001-8798-0491

https://orcid.org/0000-0002-4306-7024

https://orcid.org/0000-0002-7106-0735

https://orcid.org/0000-0002-4842-6874

\section{SUPPLEMENTARY MATERIALS}

Supplementary materials can be accessed at https://doi. org/10.5009/gnl210166.

\section{REFERENCES}

1. Mizrahi JD, Surana R, Valle JW, Shroff RT. Pancreatic cancer. Lancet 2020;395:2008-2020.

2. Paulson AS, Tran Cao HS, Tempero MA, Lowy AM. Therapeutic advances in pancreatic cancer. Gastroenterology 2013;144:1316-1326.

3. Cowan RW, Maitra A. Genetic progression of pancreatic cancer. Cancer J 2014;20:80-84.

4. Vincent A, Herman J, Schulick R, Hruban RH, Goggins M. Pancreatic cancer. Lancet 2011;378:607-620.

5. Conroy T, Desseigne F, Ychou M, et al. FOLFIRINOX versus gemcitabine for metastatic pancreatic cancer. N Engl J Med 2011;364:1817-1825.

6. Villarroel MC, Rajeshkumar NV, Garrido-Laguna I, et al. Personalizing cancer treatment in the age of global genomic analyses: PALB2 gene mutations and the response to DNA damaging agents in pancreatic cancer. Mol Cancer Ther 2011;10:3-8.

7. Kim MP, Evans DB, Wang H, Abbruzzese JL, Fleming JB, Gallick GE. Generation of orthotopic and heterotopic human pancreatic cancer xenografts in immunodeficient mice. Nat Protoc 2009;4:1670-1680.

8. Boj SF, Hwang CI, Baker LA, et al. Organoid models of human and mouse ductal pancreatic cancer. Cell 2015;160:324338.

9. Kaushik G, Ponnusamy MP, Batra SK. Concise review: current status of three-dimensional organoids as preclinical models. Stem Cells 2018;36:1329-1340.
10. Sato T, Vries RG, Snippert HJ, et al. Single Lgr5 stem cells build crypt-villus structures in vitro without a mesenchymal niche. Nature 2009;459:262-265.

11. Sato T, Stange DE, Ferrante M, et al. Long-term expansion of epithelial organoids from human colon, adenoma, adenocarcinoma, and Barrett's epithelium. Gastroenterology 2011;141:1762-1772.

12. Barker N, Huch M, Kujala P, et al. Lgr5(+ve) stem cells drive self-renewal in the stomach and build long-lived gastric units in vitro. Cell Stem Cell 2010;6:25-36.

13. Huch M, Bonfanti P, Boj SF, et al. Unlimited in vitro expansion of adult bi-potent pancreas progenitors through the Lgr5/R-spondin axis. EMBO J 2013;32:2708-2721.

14. Gao D, Vela I, Sboner A, et al. Organoid cultures derived from patients with advanced prostate cancer. Cell 2014;159:176187.

15. Huch M, Dorrell C, Boj SF, et al. In vitro expansion of single Lgr5+ liver stem cells induced by Wnt-driven regeneration. Nature 2013;494:247-250.

16. Karthaus WR, Iaquinta PJ, Drost J, et al. Identification of multipotent luminal progenitor cells in human prostate organoid cultures. Cell 2014;159:163-175.

17. Tiriac H, Bucobo JC, Tzimas D, et al. Successful creation of pancreatic cancer organoids by means of EUS-guided fineneedle biopsy sampling for personalized cancer treatment. Gastrointest Endosc 2018;87:1474-1480.

18. Morganti AG, Massaccesi M, La Torre G, et al. A systematic review of resectability and survival after concurrent chemoradiation in primarily unresectable pancreatic cancer. Ann Surg Oncol 2010;17:194-205.

19. Eloubeidi MA, Chen VK, Eltoum IA, et al. Endoscopic ultrasound-guided fine needle aspiration biopsy of patients with suspected pancreatic cancer: diagnostic accuracy and acute and 30-day complications. Am J Gastroenterol 2003;98:26632668.

20. Lacomb JF, Plenker D, Tiriac H, et al. Single-pass vs 2-pass endoscopic ultrasound-guided fine-needle biopsy sample collection for creation of pancreatic adenocarcinoma organoids. Clin Gastroenterol Hepatol 2021;19:845-847.

21. Vlachogiannis G, Hedayat S, Vatsiou A, et al. Patient-derived organoids model treatment response of metastatic gastrointestinal cancers. Science 2018;359:920-926.

22. Seidlitz T, Merker SR, Rothe A, et al. Human gastric cancer modelling using organoids. Gut 2019;68:207-217.

23. Ganesh K, Wu C, O’Rourke KP, et al. A rectal cancer organoid platform to study individual responses to chemoradiation. Nat Med 2019;25:1607-1614.

24. Driehuis E, van Hoeck A, Moore K, et al. Pancreatic cancer organoids recapitulate disease and allow personalized drug screening. Proc Natl Acad Sci U S A 2019;116:26580-26590.

25. National Institute of Environmental Health Sciences. Guid- 
ance document on using in vitro data to estimate in vivo starting doses for acute toxicity: NIH publication No. 014500. Research Triangle Park: National Toxicology Program, 2001.

26. Stein SM, James ES, Deng Y, et al. Final analysis of a phase II study of modified FOLFIRINOX in locally advanced and metastatic pancreatic cancer. Br J Cancer 2016;114:737-743.

27. Kleeff J, Korc M, Apte M, et al. Pancreatic cancer. Nat Rev Dis Primers 2016;2:16022.

28. Chen JY, Ding QY, Lv Y, et al. Slow-pull and different conventional suction techniques in endoscopic ultrasoundguided fine-needle aspiration of pancreatic solid lesions using 22-gauge needles. World J Gastroenterol 2016;22:87908797.

29. Huang L, Holtzinger A, Jagan I, et al. Ductal pancreatic cancer modeling and drug screening using human pluripotent stem cell- and patient-derived tumor organoids. Nat Med 2015;21:1364-1371.

30. Gendoo DMA, Denroche RE, Zhang A, et al. Whole genomes define concordance of matched primary, xenograft, and organoid models of pancreas cancer. PLoS Comput Biol 2019;15:e1006596.

31. Mapes BL, Bell JS, Langer LF, et al. Universal genetic and transcriptomic concordance metrics to validate patientderived tumor organoid models. Cancer Res 2020;80(16 Suppl):3908.

32. Tiriac H, Belleau P, Engle DD, et al. Organoid profiling identifies common responders to chemotherapy in pancreatic cancer. Cancer Discov 2018;8:1112-1129.

33. Broutier L, Mastrogiovanni G, Verstegen MM, et al. Human primary liver cancer-derived organoid cultures for disease modeling and drug screening. Nat Med 2017;23:1424-1435.

34. Li X, Francies HE, Secrier M, et al. Organoid cultures recapitulate esophageal adenocarcinoma heterogeneity providing a model for clonality studies and precision therapeutics. Nat Commun 2018;9:2983.

35. Baker LA, Tiriac H, Clevers H, Tuveson DA. Modeling pancreatic cancer with organoids. Trends Cancer 2016;2:176190.

36. Yachida S, Jones S, Bozic I, et al. Distant metastasis occurs late during the genetic evolution of pancreatic cancer. Nature 2010;467:1114-1117. 